\title{
A LINK BETWEEN MENGER'S THEOREM AND INFINITE EULER GRAPHS
}

\author{
L. BOZA, A. DIÁNEZ, A. MÁRQUEZ and A. QUINTERO (Sevilla)
}

\section{Introduction}

An infinite graph $G$ is usually called Eulerian if there is an Eulerian trail in $G$, i.e., a one-way or two-way infinite trail which contains all the edges of $G$. In both cases, it is obviously necessary that $V(G)$ be countable and that $G$ be, at most, two ended (in the case of one-way trail $G$ must be one ended, see [6]), which means that for each finite $K \subset G$ there is, at most, two infinite components in $G-K$ (one infinite component in the case of one ended).

It is the purpose of this note to extend the definition of an infinite Eulerian graph in such a way that the above restrictions on the number of ends are not required, but such that in the cases previously considered the "old" notion of Eulerian graph appears as a special case.

By an infinite graph we mean a graph $G$ such that its vertex set $V(G)$ is countable and the degree in every vertex is finite. In particular the edge set $E(G)$ is also countable. In other words, $G$ is a locally finite countable graph. We will use the notations and definitions of [5], except vertex instead of point and edge instead of line. And if $A$ is a set we represent its cardinal by $|A|$.

If $v$ is a vertex in a graph $G$, we denote by $\delta(v, G)$ its degree in $G$ (or by $\delta(v)$ when no confusion is possible), and by $l k(v, G)$ we denote the set of incident vertices with $v$. The set of vertices with odd degree in $G$ will be denoted by $\mathcal{O}(G)$.

If $G$ and $G^{\prime}$ are two graphs, and $v \in V(G)$ and $v^{\prime} \in V\left(G^{\prime}\right)$ are two vertices, by $G \cup_{v=v^{\prime}} G^{\prime}$ we mean the graph obtained after identifying $v$ with $v^{\prime}$ in the disjoint union of $G$ and $G^{\prime}$.

Finally, we explictly define the notion of end. A Freudenthal end of a non-compact space $X$ is an element of the inverse limit $\mathcal{F}(X)=\underline{\lim } \pi_{0}(X$ $-K$ ), where $K$ ranges over the family of compact sets of $X$ and $\pi_{0}$ stands for the set of connected components. The cardinal of $\mathcal{F}(X)$ is denoted by $e(X)$. When $X$ is a graph we can use a countable sequence $G_{1} \subseteq G_{2} \subseteq \ldots$ of finite subgraphs to obtain $\mathcal{F}(X)$ (see [1] for details). 


\section{Menger's theorems}

Definition 1. Let $G$ be an infinite connected graph and $A \subset V(G)$. $A$ is said to be Menger if there exists no subset $M \subset E(G)$ with $k<|A|$ elements such that there are $k+1$ elements of $A$ in finite components of $G-M$.

We can reformulate [3, Satz 3] in the following way.

THEOREM 2. Let $G$ be an infinite connected graph and $v \in V(G)$. Then there are $\delta(v)$ edge-disjoint 1-paths starting at $v$ if and only if $l k(v)$ is Menger.

Theorem 2 is trivially equivalent to

THEOREM 3. Let $G$ be an infinite connected graph and $A$ a finite subset of $V(G)$. Then there exist $|A|$ edge-disjoint 1-paths starting at each vertex of $A$ if and only if $A$ is Menger.

It is not difficult, by using Zorn's Lemma, to extend Theorem 2 to graphs with $\delta(v)=\infty$. But that technique does not work if we try to extend Theorem 3 to infinite subsets of $V(G)$. However, it is possible to adapt Halin's proof in order to achieve

THEOREM 4. Let $G$ be an infinite connected graph and $A \subset V(G)$, with $A$ possibly infinite. Then there exist $|A|$ edge-disjoint 1-paths starting at each vertex of $A$ if and only if $A$ is Menger. ing:

Proof. Let $\left\{H_{n}: n \in N\right\}$ be a family of finite subgraphs of $G$ satisfy-

1. $H_{i} \subset H_{i+1}$.

2. $\cup H_{n}=G$.

As a consequence of Theorem 3 , there exist $\left|A_{n}\right|$ edge-disjoint 1-paths starting at each vertex of $A_{n}=A \cap H_{n}$. Let $P_{n}$ be that set of 1-paths and let $P_{n}^{i}=P_{n} \cap H_{i}$.

Since $H_{1}$ is finite, in the sequence $\left\{P_{1}^{1}, P_{2}^{1}, P_{3}^{1}, \ldots\right\}$ there must be an element repeated infinitely many times; let $P^{1}$ be that element. In the same way, in the sequence $\left\{P_{1}^{2}, P_{2}^{2}, P_{3}^{2}, \ldots\right\}$ there must be an element repeated infinitely many times and such that the intersection of that element with $H_{1}$ agrees with $P^{1}$; let $P^{2}$ be that element. By iterating this process, we get an increasing sequence of sets of edges in $G$ that defines $|A|$ edge-disjoint 1-paths starting at each vertex of $A$.

\section{Eulerian graphs}

We call a 1-path to a graph homeomorphic to the half-line and a 2-path to a graph homeomorphic to the line. Thus, we can say that a graph $G$ is $i$-Eulerian $(i=1,2)$ if there exists a morphism $\phi: P \rightarrow G$ inducing a bijection on the edges, where $P$ is an $i$-path $(i=1,2)$ (cf. [6]). 
Following [4], we give the following definitions:

DEFINITION 5 . We define a tree $T$ to be a purely infinite tree if either it is a 1-path or an infinite tree without vertex of degree 1.

In [4] it is pointed out that 1-paths and 2-paths are the only purely infinite trees $T$ with $e(T)=1,2$. We can now give the following definition.

DEFINITION 6. An infinite connected graph $G$ is said to be Eulerian, if there exists a morphism from a purely infinite tree $T, \phi: T \rightarrow G$ inducing a bijection on the edges.

Obviously, an Eulerian graph is connected because a morphism conserves the number of connected components.

REMARK 7. As it was said above, if a graph is 1-Eulerian or 2-Eulerian, then it is Eulerian in the sense of Definition 6 . In [2] a definition of $n$ Eulerian graphs is given, namely those graphs for which there exists a morphism $\phi: W \rightarrow G$ inducing a bijection on the edges, where $W$ represents the wedge of $n 1$-way paths. It is straightforward from that definition to check that $n$-Eulerian graphs are also Eulerian in the sense of Definition 6.

In order to characterize Eulerian graphs, we are going to give the following results:

LEMMA 8. If $G$ is an infinite Eulerian graph and $G^{\prime}$ is either another infinite Eulerian graph or a finite graph with $\mathcal{O}\left(G^{\prime}\right)=\emptyset$; and $v \in V(G)$, $v^{\prime} \in V\left(G^{\prime}\right)$. Then $G \cup_{v=v^{\prime}} G^{\prime}$ is Eulerian.

Proof. The proof is a direct consequence of the definition.

Proposition 9. If $G$ is an infinite connected graph with $\mathcal{O}(G)=\emptyset$ then $G$ is Eulerian.

Proof. Let $v$ be a vertex in $G$. We denote by $E_{n}$ the subgraph induced by those vertices which are, at most, at a distance $n$ from $v$.

We are going to give a sequence of purely infinite trees rooted at $v$ and morphisms $\left\{\left(T_{1}, \phi_{1}\right),\left(T_{2}, \phi_{2}\right), \ldots\right\}$ such that $n$.

1. $T_{j} \subseteq T_{i}$ for any $i>j$ and the distance from $v$ to $T_{n}-T_{n-1}$ is, at least,

2. $\phi_{n}$ induces a bijection on the edges of $E_{n}$.

3. $\phi_{i} \mid T_{j}=\phi_{j}$ for any $i>j$.

4. $\mathcal{O}\left(G-\phi_{n}\left(T_{n}\right)\right)=\emptyset$.

5. $G-\phi\left(T_{n}\right)$ has not finite components.

Considering $T_{0}=(\{v\}, \emptyset)$, we are going to give the method to get $\left(T_{n+1}\right.$, $\left.\phi_{n+1}\right)$ from $\left(T_{n}, \phi_{n}\right)$.

If there are no edges in $E_{n+1}-\phi_{n}\left(T_{n}\right)$, then $\left(T_{n+1}, \phi_{n+1}\right)=\left(T_{n}, \phi_{n}\right)$. Otherwise, let $\{u, w\}$ be an edge in $E_{n+1}-\phi_{n}\left(T_{n}\right)$ with $u \in E_{n}$. As a consequence of Theorem 3 and the characterization of finite Eulerian graphs, 
there exists a 2-path $P$ in $E_{n+1}-\phi_{n}\left(T_{n}\right)$ containing $\{u, w\}$ such that $E_{n+1}$ $-\left(\phi_{n}\left(T_{n}\right) \cup P\right)$ has no finite components. Thus, we add two 1-paths at a vertex of $T_{n}$ which is mapped in $u$ and we extend $\phi_{n}$ to the new tree so obtained. We repeat the same process with all the edges in $E_{n+1}-\phi_{n}\left(T_{n}\right)$ in order to get $\left(T_{n+1}, \phi_{n+1}\right)$.

By using Lemma 8 we get as a corollary of Proposition 9.

COROLLARY 10. If $G$ is an infinite connected graph with an Eulerian subgraph $G^{\prime}$ such that $\mathcal{O}\left(G-G^{\prime}\right)=\emptyset$, then $G$ is Eulerian.

Now, we are going to give a generalization of Definition 1 which will be used in the characterization of Eulerian graphs.

DEFINITION 11. Let $G$ be an infinite connected graph and $A \subset V(G)$. $A$ is said to be $i$-Menger if there exists no subset $M \subset E(G)$ with $k<|A|$ elements such that there are $k-i+1$ elements of $A$ in finite components of $G-M$.

It is not difficult to check that if $T$ is a purely infinite tree then $\mathcal{O}(T)$ is 2-Menger.

Now, we can set up

THEOREM 12. An infinite connected graph $G$ is Eulerian if and only if $\mathcal{O}(G)$ is 2-Menger.

Proof. Let $G$ be an Eulerian graph. There exists a purely infinite tree $T$ and a morphism $\phi: T \rightarrow G$ such that $\phi$ induces a bijection on the edges. If $\mathcal{O}(G)$ is not 2-Menger then there exists a set $M$ of edges of $G$, with $|M|$ $<|\mathcal{O}(G)|$, such that there are $|M|-1$ vertices of $\mathcal{O}(G)$ in the union $C$ of finite components of $G-M . \phi^{-1}(C)$ is the union of finite components of $T-\phi^{-1}(M)$. As the number of elements of $\mathcal{O}(T) \cap \phi^{-1}(C)$ is at least the number of elements of $\mathcal{O}(G) \cap C$ and $\left|\phi^{-1}(M)\right|=|M|, \mathcal{O}(T)$ is not 2-Menger, but this is not possible, thus, $\mathcal{O}(G)$ must be 2 -Menger.

Conversely, let $G$ be an infinite connected graph such that $\mathcal{O}(G)$ is 2Menger. By using the same technique as in Theorem 4, we are going to give an Eulerian subgraph $G^{\prime}$ of $G$ such that $\mathcal{O}\left(G-G^{\prime}\right)=\emptyset$, thus by Corollary 10 we will achieve our result.

As in the proof of Theorem 4, let $\left\{H_{n}: n \in N\right\}$ be a family of finite subgraphs of $G$ satisfying:

1. $H_{i} \subset H_{i+1}$.

2. $\cup H_{n}=G$.

As a consequence of Theorem 4, there exist edge-disjoint 1-paths starting at each vertex of $\mathcal{O}_{n}$ and a 2-path starting at a vertex $v$ of $H_{n}$ where $\mathcal{O}_{n}$ $=\mathcal{O}(G) \cap H_{n}$. Let $P$ be the subgraph of $G$ defined by union of those 1-paths and the 2-path; it is clear that $\mathcal{O}(G-P)=\emptyset$. Now, we are going to "grow" $P$ in order to get an Eulerian subgraph $G^{\prime}$ with $P \subseteq G^{\prime}$.

Let $P_{n}$ be the union of the 2-path with the 1-paths starting at $\mathcal{O}_{n}$. Since $\mathcal{O}\left(H_{n}-P_{n}\right)=\emptyset$, it is possible to apply [7, Theorem 2.2] in order to get an 
Eulerian graph $D_{n}$ such that $P_{n} \subseteq D_{n}$. Basically, $D_{n}$ is obtained by adding to $P_{n}$ either cycles or 2-paths in such a way that we get a connected graph. As far as we keep adding only either Eulerian graphs - 2-paths - or cycles, it is possible to apply Lemma 8 to assure that $D_{n}$ is Eulerian.

Now let $D_{n}^{i}=D_{n} \cap H_{i}$. And, as in Theorem 4, we know that in the sequence $\left\{D_{1}^{1}, D_{2}^{1}, D_{3}^{1}, \ldots\right\}$ there must be an element repeated infinitely many times; let $D^{1}$ be that element. In the same way, in the sequence $\left\{D_{1}^{2}, D_{2}^{2}, D_{3}^{2}, \ldots\right\}$ there must be an element repeated infinitely many times and such that the intersection of that element with $H_{1}$ agrees with $D^{1}$; let $D^{2}$ be that element. By iterating this process, we get an increasing sequence $D^{1} \subseteq D^{2} \subseteq \ldots$ of Eulerian subgraphs, and so $G^{\prime}=\bigcup D^{n}$ is trivially Eulerian. Now it only remains to apply Corollary 10 to assure that $G$ is Eulerian. 


\section{References}

[1] R. Ayala, E. Domínguez, A. Márquez and A. Quintero, Proper homotopy classification of graphs, Bull. London Math. Soc, 22 (1990), 417-421.

[2] L. Boza, A. Diánez, A. Márquez and A. Quintero, A generalization of eulerian graphs, Preprint.

[3] R. Halin, Über Trennende Fckenmengen in Graphen und den Mengerschen Satz, Math. Ann., 157 (1964), 34-41.

[4] R. Halin, On the notion of infinite hamiltonian graph, Journal of Graph Theory, 7 (1983), 437-440.

[5] F. Harary, Graph Theory, Addison Wesley (Reading, Mass., 1969).

[6] O. Ore, Theory of Graphs, Volume 38 of Colloquium Publications (AMS, 1962).

[7] G. Sabidussi, Infinite Euler graphs, Canadian Journal of Mathematics, XVI (1964), $821-838$.

(Received May 29, 1995; revised September 25, 1995)

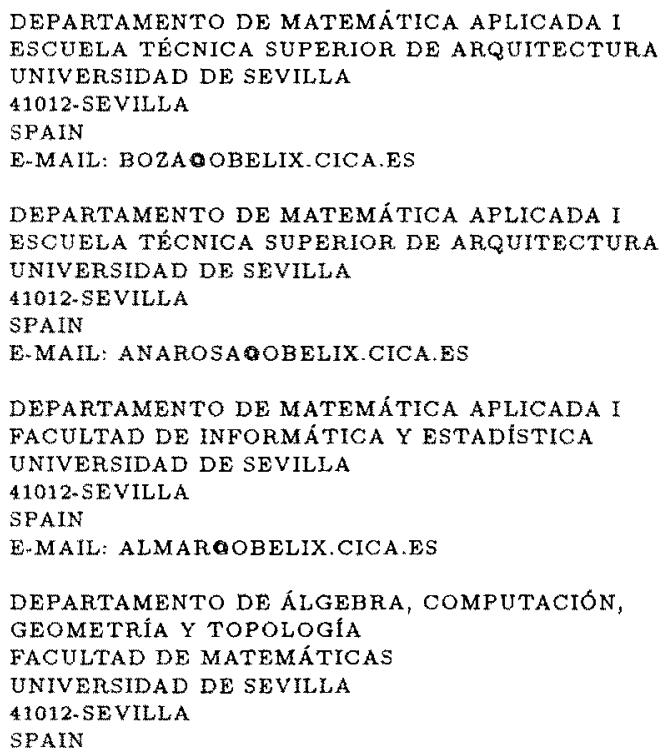

